\title{
A Novel Super Resolution Algorithm based on Fuzzy Bicubic Interpolation Algorithm
}

\author{
A. Geetha Devi ${ }^{1}$, T. Madhum ${ }^{2}$ and K. Lal Kishore ${ }^{3}$ \\ Dept., of ECE, \\ ${ }^{1} P V P$ Siddhartha Institute of Technology, Vijayawada, India \\ ${ }^{2}$ Swarnandra Institute of Engg., \& Tech., Narasapuram, India \\ ${ }^{3}$ Jawaharlal Nehru Technological University, Ananthapur, Andhra Pradesh, \\ India \\ geetha.agd@gmail.com,tennetimadhu@yahoo.com
}

\begin{abstract}
Super Resolution (SR) reconstruction is a procedure of attaining the High resolution $(H R)$ images from a set of noisy and blurred low resolution (LR) observations. In this paper a novel SR algorithm is proposed by developing a fuzzy based Bicubic interpolation algorithm to improve the resolution of the image. The algorithm significantly improves the resolution, quality and enhances the color information of the reconstructed image when compared with the other existing methods.
\end{abstract}

Keywords: Super Resolution, Image Interpolation, Adaptive sharpening filter, steering kernel regression

\section{Introduction}

The advent of new technologies and development of computer vision applications over the past two decades demand for high resolution and high quality images for their efficient functioning and better quality. In fact the demand is increasing exponentially. The quality of image information determines the efficiency and effectiveness of applications such as medical imaging, remote sensing, HDTV (High Definition Television), Video Surveillance, Video conferencing etc. Hence, high resolution images are required to improve the efficacy of these systems. Although High Precision optics and sensors will produce high resolution images, their cost is very high. Hence, efforts are made to improve the resolution of images acquired from low precision, low cost image acquisition equipment by using signal processing methods known as the Super Resolution(SR) image reconstruction technique and is defined as the method of obtaining High Resolution [HR] images from a set of noisy and blurred low resolution observations. The generalized block diagram of the super resolution image reconstruction is depicted in Figure 1.

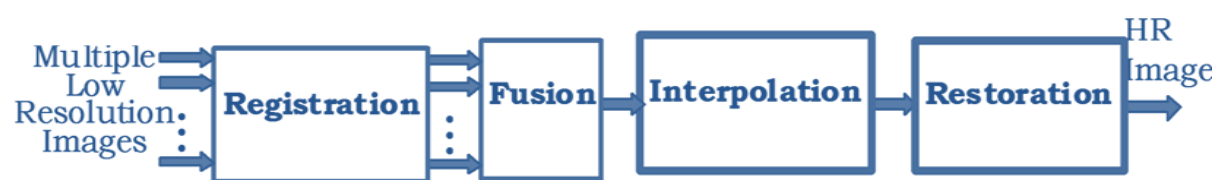

Figure 1. The Generalized Block Diagram of Super Resolution

There are two types of SR image reconstruction: single image SR or multi image SR. Single image SR techniques require a large amount of data base for training where as the multiple image SR Methods deal with the inverse problem [1]. In multiple SR reconstruction technique, the low resolution (LR) images are obtained with a low 
resolution camera or sensor operated from different viewpoints, at different times or with the use of cameras having different resolution. The low resolution observations can be formulated as

$$
y_{c, i}=D H F_{c, i} X_{c}+N_{c, i} \quad c=\mathrm{R}, \mathrm{G}, \mathrm{B} \quad \& \quad i=1,2,3 \ldots \ldots \ldots \ldots . \mathrm{N} .
$$

Where $\mathrm{N}$ is the number of low resolution observations made, $X c$ is the $c^{\text {th }}$ colour component of unknown High resolution image, $y_{c, i}$ is the $i^{\text {th }}$ low resolution image of the $X c$, $\mathrm{D}$ is the down sampling matrix, $\mathrm{H}$ is the point spread function of the blur operator, $\mathrm{F}_{\mathrm{c}, \mathrm{i}}$ is the warping matrix and $\mathcal{N}_{c, i}$ is the additive noise. The LR images so obtained must be brought onto a common geometrical plane to improve the resolution and the process is known as image registration [2]. Image registration techniques such as point based, feature based and area based image registration techniques are studied [3]. Feature based image registration \& analysis is found to be more suitable for medical imaging applications [18]. Further, as more pixel details are available in a number of low resolution images due to the sub pixel shift among them, all the registered LR images are to be fused into a single HR grid and interpolated to improve the resolution of the image [23].The Singular Value Decomposition (SVD) technique is mainly utilized in facial feature extraction, recognition and in many SR reconstruction problems for effective identification of features in LR images when compared to other fusion algorithms [13] and hence is utilized in the proposed algorithm.

In SR image reconstruction algorithms image up sampling is essential to improve the resolution of the image. Image up sampling algorithms employ spatial interpolation algorithms [5-9] to enlarge the spatial resolution of the LR images and to compute the missing information from spatially adjacent data. Conventional interpolation methods like nearest neighborhood interpolation and bilinear interpolation methods exhibit high computational simplicity but face severe blurring problems particularly at the edge information. On the other hand, Bicubic and Spline interpolation methods [24] are computationally complex, provide better results but still exhibit some degree of blurness. To overcome these problems, a fuzzy based Bicubic interpolation algorithm is developed and applied to SR algorithm to reconstruct a HR image at a resolution which is pleasing to the viewer without any visual artifacts. The fused image is applied to fuzzy based Bicubic image interpolation algorithm to improve the resolution. The restoration method has to be applied depending on the type of additive noise present in the LR images. Therefore, Adaptive sharpening filter based restoration [10] will be applied in case of Gaussian noise, Adaptive Geometric Feature based Filter (AGFF) technique of restoration [11] in case of impulse noise and the combination of these two algorithms for mixed noise environment.

The proposed method is compared with the other available methods [12-13] using different comparison metrics like mean, standard deviation, entropy, PSNR, correlation coefficient and Universal Image Quality Index (UIQI) [17]. The rest of the paper is organized as follows: Section 2 explores the fuzzy Bicubic interpolation algorithm. Section 3 describes the proposed algorithm. Section 4 discusses the results and concluding remarks are specified in section 5 .

\section{Fuzzy Bicubic Interpolation}

The concept of Bicubic interpolation is described in the following section before exploring the proposed algorithm.

\subsection{Bicubic Interpolation}

In image processing, bicubic interpolation is often preferred over the nearest neighborhood or bilinear interpolation [10] when speed of operation is not a matter. Bi- 
cubic Interpolation is an application of cubic interpolation on 2-D and attempts to reconstruct the exact surface by extracting the sixteen pixels of image information from the closest $4 \times 4$ neighborhood as shown in the Figure.2.

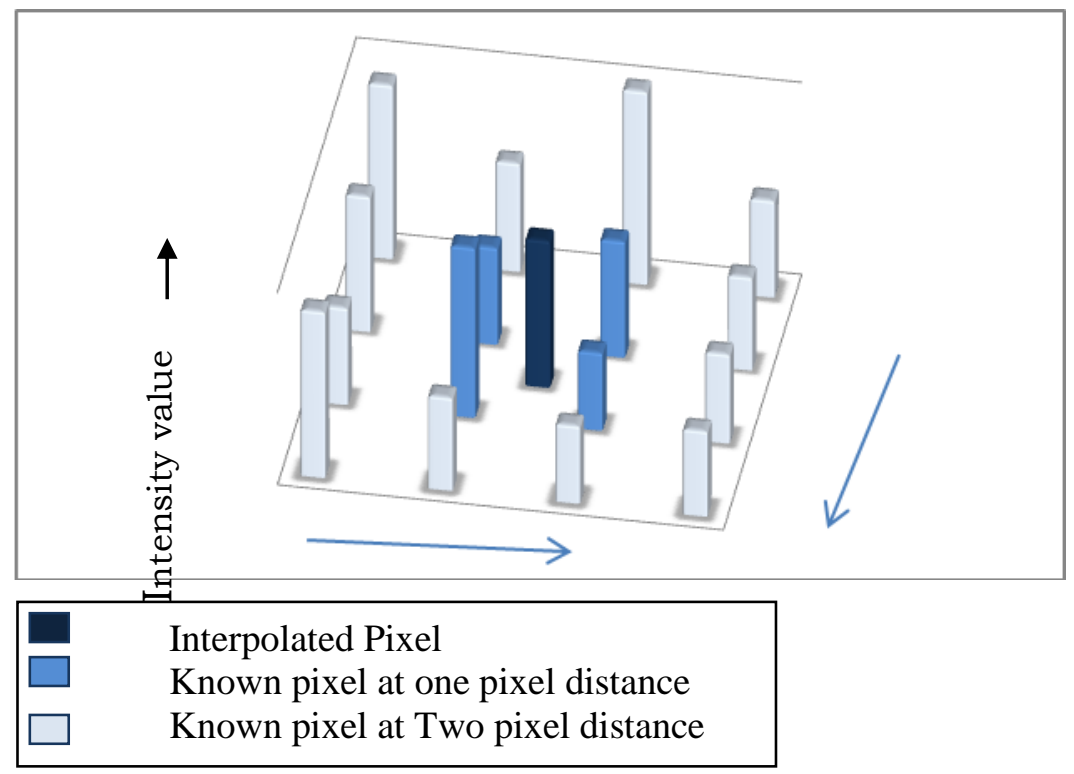

Figure 2. Pictorial Representation of Bicubic Interpolation

The bi-cubic interpolation kernel is

$$
f(x)= \begin{cases}(a+2)|x|^{3}-(a+3)|x|^{2}+1 & \rightarrow 0<|x|<1 \\ (a)|x|^{3}-5 a|x|^{2}+8 a|x|-4 a & \rightarrow 1<|x|<2 \\ 0 & \rightarrow \text { elsewhere }\end{cases}
$$

Here, a higher weight is assigned to the closer pixels. Bi-cubic interpolation produces noticeably sharper images than the nearest neighborhood and bilinear interpolation techniques with negligible artifacts and is perhaps the ideal combination of processing time and output quality. For this reason it is a standard in many image editing programs, printer drivers and in-camera interpolation. The Bicubic interpolation is visualized as 1-D cubic interpolation in two dimensions i.e., row wise and column wise or vice versa. 1-D Cubic Convolution Interpolation [9] considers four grid points to evaluate the interpolation function i.e., two grid points on either side of the point under consideration. For Bi-cubic interpolation the number of grid points needed to evaluate the interpolation function is 16, two grid points on either side of the pixel under consideration for both horizontal and vertical directions. The grid points needed in one-dimension and twodimension cubic convolution interpolation are shown in Figure.3

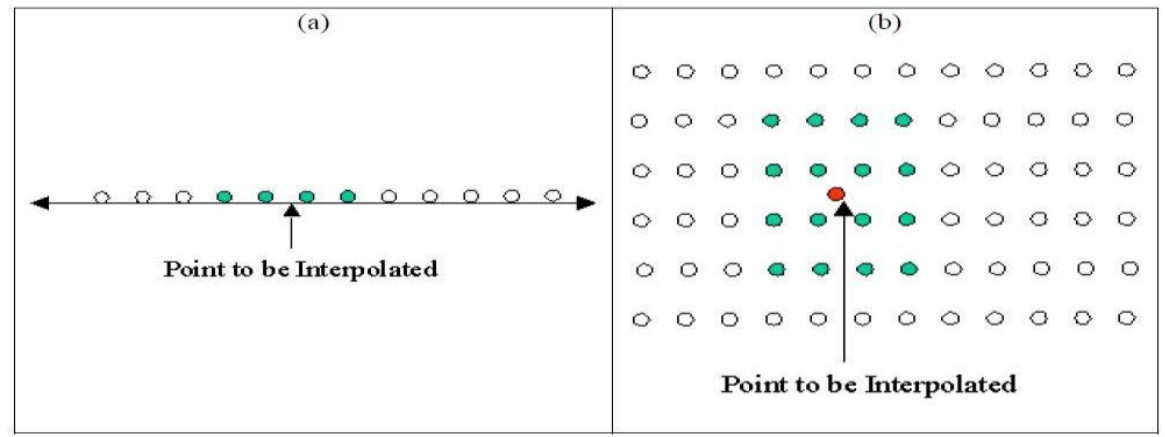

Figure 3. Grid Points in 1-D \& 2-D Cubic Interpolation 
The red green circles are the grid points to be considered to calculate the interpolated value at the position shown by arrow mark.

\subsection{Fuzzy Bi-cubic Interpolation}

The fuzzy image interpolation implementation combines a fuzzy inference system and an image interpolation technique. It utilizes a dynamic mask combined with a sophisticated averaging fuzzy algorithm in a cubic manner to perform image scaling operation. The dynamic mask is applied two times in horizontal and vertical directions or vice versa. The functions that contribute to the final interpolated image are the areas of the input pixels, overlapped by a dynamic mask, and the difference in intensity between the input pixels. These two functions are applied with Fuzzy if-then rules to carry out the interpolation task. This algorithm can interpolate both grey scale and color images to any scaling factor. The aim of the proposed approach is to maximize the smoothness of the interpolated image by preserving the continuous and sharp edges. A maximum of four contributing neighboring pixels is chosen in either direction to reduce the computational burden.

Three steps are required to describe the overall scaling process. First, the output image is constructed through undefined pixel intensity values, according to the magnification factor. Subsequently, the area of the dynamic mask is determined, as its value depends on the scaling factor. Next, each output pixel is mapped onto the input image based on the dimensions of the mask applied. The overlapped input pixels could be one, two or four in 2-D. But, in 1-D, the over lapped pixels are either 1 or 2 . For each overlapped input pixel, area functions that describe the area coverage from the mask and the differences in intensity of the overlapped input pixels are computed. Finally, these two functions are applied to the Fuzzy Inference System (FIS) is to extract the proportional input pixel weights that will determine the intensity of the output pixel. The flow chart of the proposed algorithm is depicted in the Figure 4.

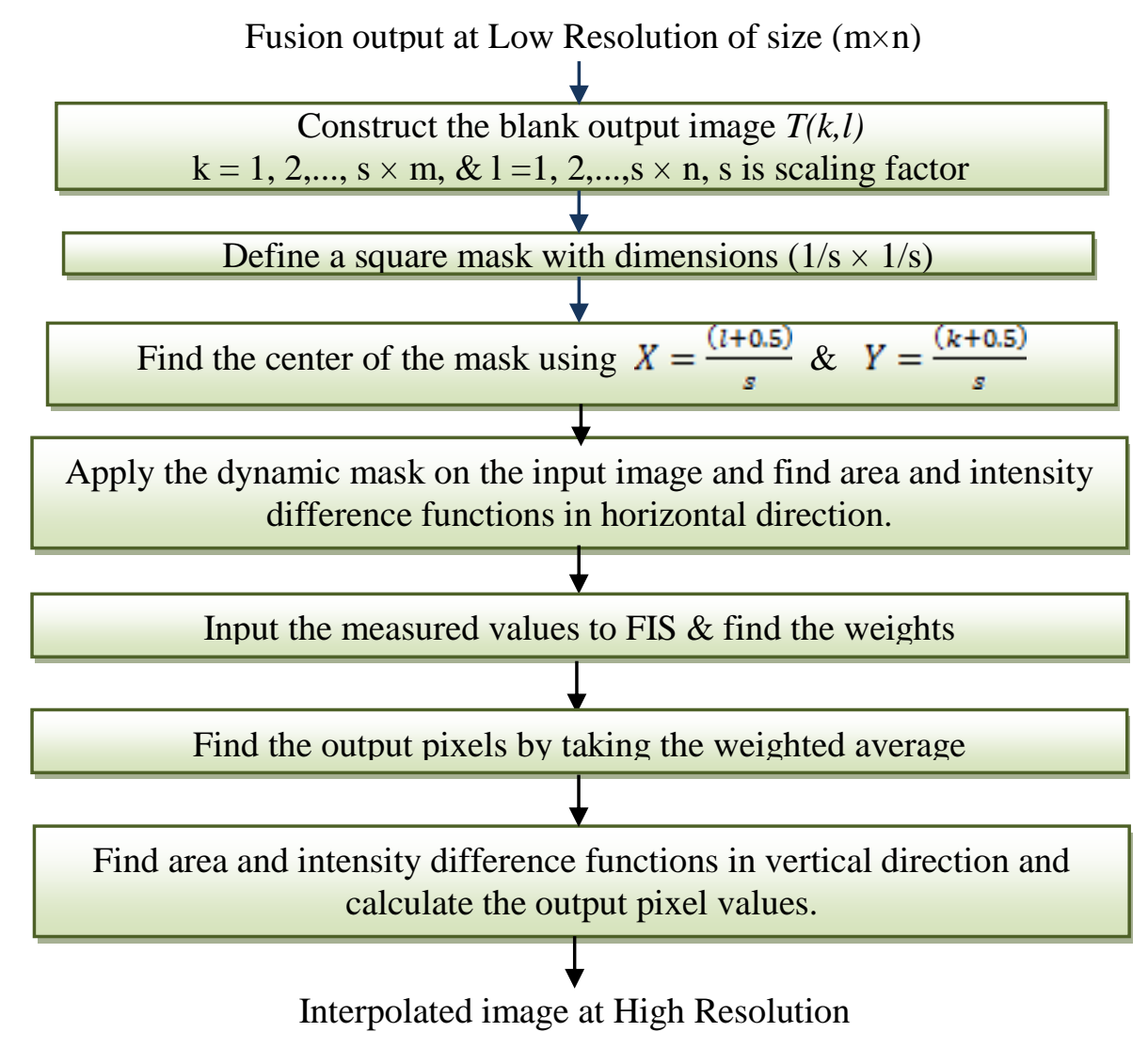

Figure 4. Flow Chart of the Proposed Fuzzy based Bicubic Interpolation 
2.2.1 Construction and Application of Dynamic Mask: Let $S(i, j)$ be the input image with dimensions $m \times n$ and scale factor $\mathrm{s}$. For the scaling-up process, first, the output image $\mathrm{T}(\mathrm{k}, \mathrm{l})$ is constructed, where $\mathrm{k}=1,2, \ldots, \mathrm{s} \times \mathrm{m}$, and $\mathrm{l}=1,2, \ldots, \mathrm{s} \times \mathrm{n}$. For a fractional scaling factor, a floor operation to the dimensions of the output image is applied. The intensity values of all pixels in $\mathrm{T}$ are left undefined. The square mask dimensions are defined as

$$
M_{\text {height }}=\frac{1}{s} \text { and } M_{\text {length }}=\frac{1}{s}
$$

The mask dimensions are dynamic, depending on the scaling factor. This feature guarantees that after the interpolation, no input area is used twice or more in the scaling process [14]. Next, each output pixel is mapped onto the input image plane. The coordinates of the center of the mapped output pixel are calculated using the following equations to allocate the the center of the applied mask in Cartesian plane as

$$
X=\frac{(l+0.5)}{s} \quad \text { and } Y=\frac{(k+0.5)}{s}
$$

Where $\mathrm{k}=1,2, \ldots, \mathrm{s} \times \mathrm{m}$, and $\mathrm{l}=1,2, \ldots, \mathrm{s} \times \mathrm{n}$, assuming that each pixel has height and width equal to one. The application of the dynamic mask is illustrated in the Figure 5.

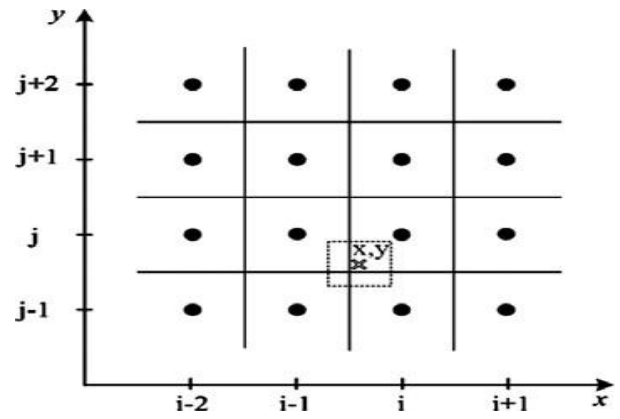

Figure 5. Application of Dynamic Mask on the Input Image [14]

Once the mask is applied, there are basically three possible conditions. The first is that the mask entirely lies within one pixel of the input image. The second is that the projection partially lies in two input pixels, and the third is that it lies in four input pixels. In the latter two cases, the pixel that is mainly covered from the mask is characterized as the main pixel. In case of applying the mask in one direction at a time, the mask can cover either one or two pixel areas at a time.

2.2.2. Area and Intensity Functions Calculation: To determine the sub-area functions for each overlapped input pixel, the area equations are formulated in the Cartesian coordinates. The area functions in the horizontal direction are formulated as

$$
\begin{aligned}
& f_{(i, j)}(x, y)=\left(y-j+\frac{1}{2 s}+0.5\right) \times\left(x-i+\frac{1}{2 s}+0.5\right) \\
& f_{(i, j-1)}(x, y)=\left(y-j+\frac{1}{2 s}+1.5\right) \times\left(x-i+\frac{1}{2 s}+0.5\right)
\end{aligned}
$$

The pixels at positions $(i, j+1) \&(i, j-2)$ are applied with the area functions defined by Eq. $5 \& 6$ respectively. As the dynamic mask cannot cover these pixels, they should be weighted proportionally with their adjacent pixels in horizontal direction. The differences in intensity functions can be easily determined by

$$
\begin{aligned}
& g 1=0 \\
& g 2=|S(i, j+1)-S(i, j)|
\end{aligned}
$$




$$
\begin{aligned}
& g 3=|S(i, j-1)-S(i, j)| \\
& g 4=|S(i, j-2)-S(i, j)|
\end{aligned}
$$

The area functions $f$ in the vertical direction are formulated as

$$
\begin{array}{r}
f_{(i, j)}(x, y)=\left(y-j+\frac{1}{2 s}+0.5\right) \times\left(x-i+\frac{1}{2 s}+0.5\right) \\
f_{(i-1, j)}(x, y)=\left(y-j+\frac{1}{2 s}+0.5\right) \times\left(x-i+\frac{1}{2 s}+1.5\right)
\end{array}
$$

The pixels at $(i+1, j) \&(i-2, j)$ are applied with the area functions defined by Eq. $11 \&$ 12 respectively. Here also, as the dynamic mask cannot cover these pixels, they should be weighted proportionally with their adjacent pixels in vertical direction. The differences in intensity functions ' $g$ ' can be easily determined by

$$
\begin{aligned}
& g 1=0 \\
& g 2=|S(i+1, j)-S(i, j)| \\
& g 3=|S(i-1, j)-S(i, j)| \\
& g 4=|S(i-2, j)-S(i, j)|
\end{aligned}
$$

Where $S(i, j)$ is the main pixel under consideration. Similar equations are used to represent $f(x, y)$ and $g(x, y)$ functions for the cases of one or two overlapped input pixels by the applied mask.

The value of the output pixel is derived by the weights calculated from the area and intensity functions. The area and difference in intensity components are applied as two separate inputs to a fuzzy (Mamdani type) inference system. The system consists of four Gaussian curve membership functions (GCMFs) for the area components, five GCMFs for the difference in intensity of the input pixels, and five GCMFs for the weight outputs. The pixel intensity values range between zero and one after the normalization process. The output variable indicates the proportional weight of each contributing pixel. Although there are different membership functions available the Gaussian membership function is chosen due to its smoothness and non-zeroness at all points. The number of membership functions is chosen to be only five for the area coverage and the difference in intensity input variable, respectively, to meet an adequate overall speed. The membership functions of the FIS are illustrated in the Figure 6.

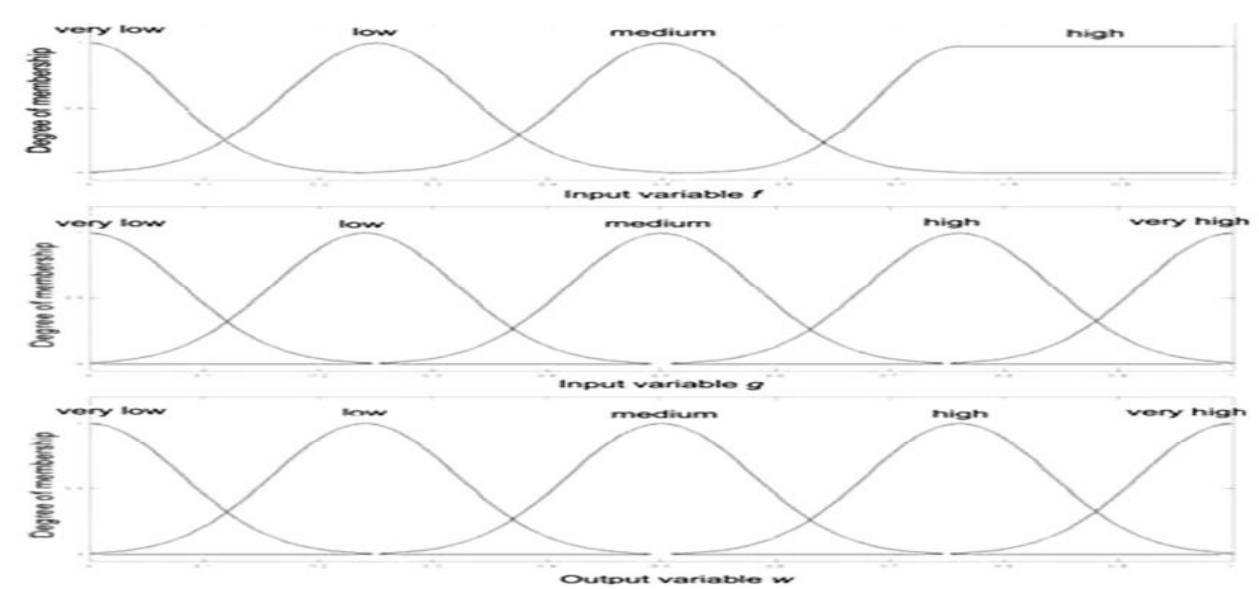

Figure.6: Graphical Representation of Input and Output Membership Functions 
The Gaussian membership function is given by

$$
f(x, \sigma, m)=e^{\frac{-(x-m)^{2}}{\mathbf{n} \sigma^{2}}}
$$

where $\sigma$ determines the shape of the curve (standard deviation), and $m$ (mean) locates the center of the curve. Defuzzification is carried out using centroid.

The two inputs are cross connected to the output through a set of 16 if-then rules and are presented in Table 1 . Here $f$ represents coverage area, $g$ represents difference in intensity and w represents output of the FIS.

Table 1. Rules Applied to the FIS

\begin{tabular}{|l|l|l|l|}
\hline S.No & If f is & and g is & then w will \\
be
\end{tabular}

The main pixel, which is mainly covered by the mask, has no difference in intensity, the output is produced through the direct de-fuzzification of the area component and is expressed through the last rule in Table 7.1.

Fuzzy rules are separately applied to each overlapped input pixel to have the required output weight for each contributing overlapped $S(i, j)$ input pixel. Finally, the output pixel intensity is given by

$$
T(k, l)=\sum_{n=0}^{1} \sum_{m=0}^{1} W_{i-n, j-m} \times S(i-n, j-m)
$$

where $\mathrm{Wi}, \mathrm{j}$ represents the final normalized weights extracted from the FIS for each equivalent intensity $S(i, j)$ of the input pixel.

This approach presents effective performance in edge regions while preserving the sharpness of the image, without any additional processing. Although choosing the inference by using Gaussian membership functions renders the process more time demanding, the results are superior to those involving linear membership functions such as triangular or trapezoidal ones. However, the Gaussian membership function process takes a lot of time to generate the results as the delay depends upon the resolution of the input image taken. 


\section{Proposed Super Resolution Algorithm}

The proposed SR algorithm using the fuzzy based interpolation is depicted in Figure.7.

Low Resolution Frames of the intended object

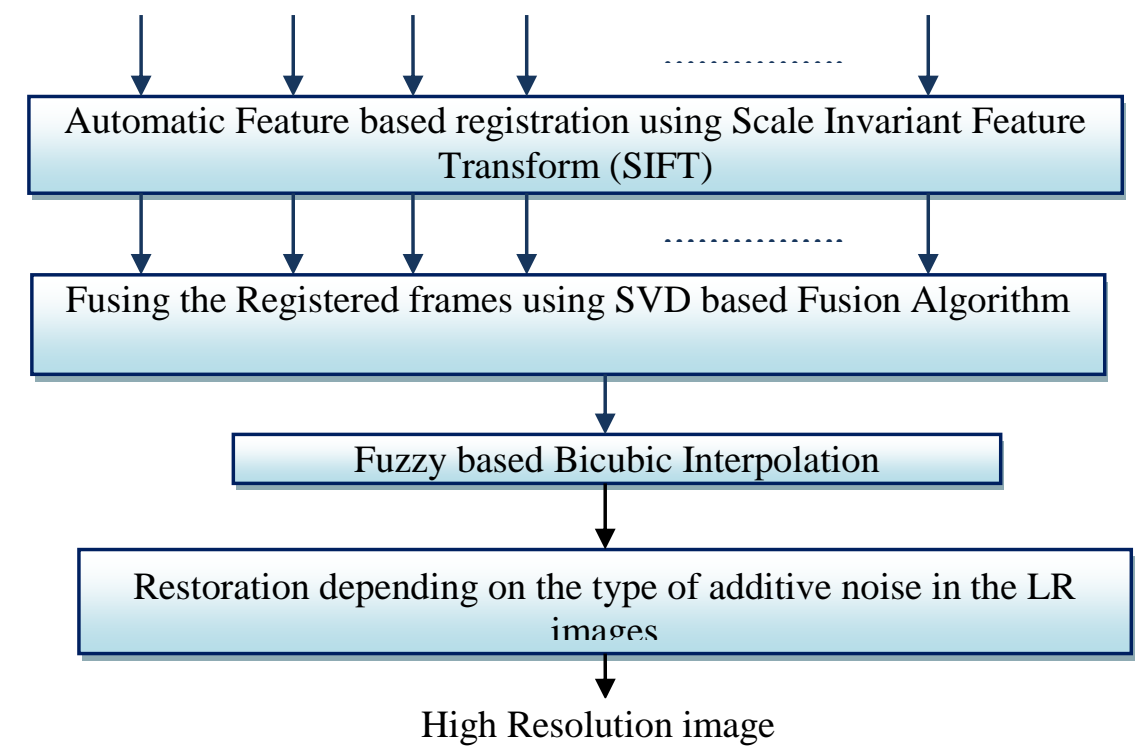

Figure 7. Block Diagram of the Proposed SR Algorithm
Using Fuzzy based Bicubic Interpolation

\subsection{Automatic feature based Registration using SIFT:}

Registration is the process of bringing all the shifted versions of low resolution images into a single plane with respect to a reference image. Feature based registration comprises of feature detection, feature matching, optimum transformation and up-sampling and provides better results in many applications. Automatic Feature based registration using Scale Invariant Feature Transform [15][18-20] is used for registration. These algorithms extract the distinct features from different images and are called as control points. The location and scale of these control points are determined by Scale Invariant Feature Transform (SIFT). The detection of location of the control points (SIFT keys) should be invariant to scale change, translation or rotation, since these keys can be repeatedly assigned to different views of the same object. The detection of the SIFT key locations is accomplished by searching for stable features across all possible scales, using a continuous function of scale known as scale space and can be determined only by Gaussian function [15]. These SIFT keys are obtained by using the Difference of Gaussian (DoG) filter [20].Hence, the scale space of an image is defined as a function $M(x, y, \sigma)$, which is produced by convolving a variable scale Gaussian function $G(x, y, \sigma)$ with the input image $I(x, y)$.

$$
M(x, y, \sigma)=G(x, y, \sigma) * I(x, y)
$$

Where $*$ is the convolution operator, $\mathrm{x}, \mathrm{y}$ are the coordinators and $\sigma$ is standard deviation of Gaussian Function defined by

$$
G(x, y, \sigma)=\frac{1}{2 \pi \sigma^{2}} e^{-\left(x^{2}+y^{2}\right) / 2 \sigma^{2}}
$$


The DoG (Difference of Gaussian) function DoG $(\mathrm{x}, \mathrm{y}, \sigma)$ is computed by taking the difference of two Gaussian functions with different standard deviations, separated by a constant multiplicative factor $k$. i. e.

$$
\begin{gathered}
\operatorname{DoG}(x, y, \sigma)=(G(x, y, k \sigma)-G(x, y, \sigma)) * I(x, y) \\
=M(x, y, k \sigma)-M(x, y, \sigma)
\end{gathered}
$$

The normalized Laplacian of Gaussian (LoG) operator, $\sigma^{2} \nabla^{2} G$ [25], which has close approaximation to the DoG is required for the scale invariance. It is found that the maxima and minima of $\sigma^{2} \nabla^{2} G$ will produce the most stable image features. The relationship between the DoG function and Laplacian function $\sigma^{2} \nabla^{2} G$ can be understood from the heat diffusion equation.

$$
\begin{gathered}
\frac{\partial G}{\partial \sigma}=\sigma \nabla^{2} G \begin{array}{c}
\left.\sigma \nabla^{2} G=\frac{\partial G}{\partial \sigma}=\frac{(G(x, y, k \sigma)-G(x, y, \sigma))}{k \sigma-\sigma}\right) \\
G(x, y, k \sigma)-G(x, y, \sigma) \approx(k-1) \sigma^{2} \nabla^{2} G
\end{array}
\end{gathered}
$$

The DoG function has scales differing by a constant factor (k-1) over all scales and therefore does not influence extreme location. The approximation error goes to zero if $\mathrm{k}=1$ but the approximation has no impact on the extreme detection or localization in practice. The maxima and minima are obtained by computing the pixel to its 26 neighbours in $3 \times 3$ regions at the current and adjacent scales. Maxima and Minima are selected only if it is larger than all of these neighbours or smaller than all of them. Most of the sample points will be eliminated in the first few checks. Hence the cost is reasonably low [10].

Based on each gradient direction of each key point, the location will be given in one or more orientations. Once a key point candidate has been found, the next step is to obtain a detailed fit to the nearby data for location, scale and ratio of principal curvatures. This information allows points to be rejected that have low constant or poorly localized along the edge. After assigning a consistent orientation to each key point based on the image local properties, the key point descriptor can be represented relative to this orientation and therefore achieve invariance to image rotation.

Towards a more stable local orientation, the scale of the key point is used to select the Gaussian smoothed image with the closest scale, so that all computations are performed in a scale invariant manner. For each image sample $M(x, y)$ at this scale, the gradient magnitude $\mathrm{m}(\mathrm{x}, \mathrm{y})$ and orientation $\theta(x, y)$ are pre computed using pixel differences [18].

$$
\begin{gathered}
m(x, y)=\sqrt{(M(x+1, y)-M(x-1, y))^{2}+(M(x, y+1)-M(x, y-1))^{2}} \\
\theta(x, y)=\tan ^{-1} \frac{M(x y+1)-M(x, y-1)}{M(x+1, y)-M(x-1, y)}
\end{gathered}
$$

An orientation histogram is formed from the gradient directions of sample points with in a region around the key point. The orientation histogram has 36 bins covering 360 degrees. Each sample added to the histogram is weighted by its gradient magnitude and by a Gaussian weighted circular window with a $\sigma$ that is 1.5 times that of scale of the key point. The peaks in the orientation histogram correspond to the dominant directions of local gradients. First the highest peak in the histogram is detected and then any other local peak value that is within $80 \%$ of the highest value is used to create a key point with that same orientation. Therefore, for locations with multiple peaks of similar magnitude, there will be multiple key points created at the same location and scale but with different orientations. Although, only around $15 \%$ of the points are assigned multiple orientations, they will significantly contribute to the stability of matching. Finally a parabola is fit to the three histogram values closest to each peak value to interpolate the peak position for better accuracy. 
Feature matching establishes the correspondence between the detected features of an image. The regular approach is to build local descriptors around the feature point and then match them. This is a very crucial step because the precision of the transformation depends on the accuracy in the correct match identification. Euclidian distance matching, invariant moment and nearest neighbour based matching are the usual methods of feature matching [14].

RANdom SAmpling Consensus (RANSAC) [13], a strong feature estimator, classifies the matching features into inliers and outliers, where inliers are the features that hold on the model while the outliers will not. The RANSAC algorithm starts by randomly selecting the set of corresponding points. For each possible set of four key points in the reference image and the corresponding match in the target image, a mapping transform is found. The transformation matrix is estimated using

$$
\left[\begin{array}{l}
x^{y} \\
y^{y} \\
1
\end{array}\right]=T\left[\begin{array}{l}
x \\
y \\
1
\end{array}\right]
$$

Where $(x, y) \leftrightarrow\left(x^{\prime}, y^{\prime}\right)$ are the coordinates of the matching points in the target image and reference image respectively and $\mathrm{T}$ is the transformation matrix. The symmetric transfer error $d\left[(x, y), T\left(x^{\prime}, y^{\prime}\right)\right]^{2}+d\left[\left(x^{\prime}, y^{\prime}\right), T(x, y)\right]^{2}$ is calculated for every matching point and the inliers that are less than the threshold are counted. Here $d\left[(x, y), T\left(x^{\prime}, y^{\prime}\right)\right]$ is the Euclidian distance between pixel points with coordinates $(x, y)$ and $\left(x^{\prime}{ }_{3} y^{\prime}\right)$. The same procedure is applied to the rest of key points in the reference image and the spatial coordinates of transformed key points are compared with the coordinates of the respective key points in the target image. This allows to find the number of key point pairs that fit in the model with certain tolerance. The optimal model is one that supports the maximum number of key point pairs within the transform model. After finding the optimal value, the model will transform the target image into the reference image, so that the corresponding points are partially coincident in both the images.

\subsection{Singular Value Decomposition (SVD) based Fusion}

The Singular Value Decomposition (SVD) technique is mainly utilized in facial feature extraction and recognition and is used in many SR reconstruction problems for effective identification of features in LR images. The singular value decomposition (SVD) technique is widely used in signal processing, pattern recognition and image compression algorithms [4].

The SVD of any image of size mxn is represented by

$$
I=U S V^{T}
$$

where $U$ and $V$ are orthogonal to each other. The columns of $U$ are Eigen values of $I^{*} I^{T}$ and the columns of $V$ matrix are Eigen values of $I^{T * I}$. $U$ is called left singular vector matrix and $V$ is called right singular vector matrix. The diagonal elements of the $n x n$ matrix $S$ represent the intensity information of $I$ and is called singular value matrix. The grey scale representation of any image is a two dimensional matrix and can be decomposed into SVD.

The highest singular value has the greatest amount of input information and the highest SVD lies at the upper left corner of the S matrix. Hence, let us represent the two input images $I_{1}$ and $I_{2}$ in the form of SVD as

$$
I_{1}=V_{1} S_{1} V_{1}^{T} \text { and } I_{2}=V_{2} S_{2} V_{2}^{T}
$$


For the colour images, decomposition is performed in each color plane separately. Let the maximum values of $S_{1}$ and $S_{2}$ are $\beta_{1 \max }$ and $\beta_{2 \max }$ respectively. If $\beta_{1 \max }>\beta_{2 \max }$ then $S_{1}$ is used in the reconstruction of the fused image otherwise $S_{2}$ is used.

Where $S_{\max }=S_{1}$ if $\beta_{1 \max }>\beta_{2 \max } \&$

$$
I_{\text {fused }}=V_{2} S_{\max } V_{2}^{T}
$$

$S_{\max }=S_{2}$ if $\beta_{2 \max }>\beta_{1 \max }$

\subsection{Restoration}

Restoration is the process of removing noise and blur from the degraded image. Usually the images are corrupted by Gaussian noise or impulse noise or both (Mixed noise). The selection of the restoration algorithm has to consider the type of noise that has been added to the images. Hence in the proposed algorithm adaptive sharpening filter based restoration is applied in case of Gaussian noise [10], AGFF technique of restoration in case of impulse noise [11] and the combination of these two algorithms for mixed noise environment [16].

\section{Results}

Two LR datasets corrupted by Gaussian noise (Figure $8 \& 9$ ) are applied to the proposed SR reconstruction algorithm. Adaptive sharpening filter restoration is applied to the SR econstruction algorithm. The result obtained by the proposed algorithm is shown in Figure 12 and is compared with the reconstructed images obtained with Blind deconvolution based SR [12] and Adaptive sharpening filter based SR [13] as shown in Figure $10 \& 11$ respectively for data set -1 . For data set-2, Figure.15 provides the reconstructed image of the proposed algorithm which is compared with the reconstructed images obtained with Blind deconvolution based SR [12] and Adaptive sharpening filter based SR [13] as shown in Figure.13 \& 14 respectively. Table.2 provides the comparison parameters for the two datasets. From the results, a maximum of $2 \mathrm{~dB}$ in PSNR value is improved in the developed algorithm along with an increment of 0.03 in C.C., 0.02 in UIQI, 2 in mean value, 0.3 in Entropy, 1 S.D

Table 2. Performance Evaluation of Proposed Algorithm

\begin{tabular}{|c|c|c|c|c|c|c|}
\hline \multirow[b]{2}{*}{ 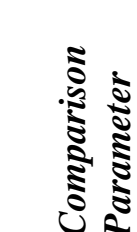 } & \multicolumn{3}{|c|}{ Dataset1 } & \multicolumn{3}{|c|}{ Dataset2 } \\
\hline & $\begin{array}{l}\text { Proposed } \\
\text { Algorithm }\end{array}$ & $\begin{array}{l}\text { SR using } \\
\text { Blind } \\
\text { Deconvolution }\end{array}$ & $\begin{array}{l}\text { SR using } \\
\text { Adaptive } \\
\text { sharpening } \\
\text { Filter }\end{array}$ & $\begin{array}{l}\text { Proposed } \\
\text { Algorithm }\end{array}$ & $\begin{array}{l}\text { SR using } \\
\text { Blind } \\
\text { deconvolution }\end{array}$ & $\begin{array}{l}\text { SR using } \\
\text { Adaptive } \\
\text { sharpening } \\
\text { Filter }\end{array}$ \\
\hline PSNR & 44.783 & 42.132 & 42.456 & 51.517 & 49.234 & 30.9876 \\
\hline C.C. & 0.8001 & 0.8025 & 0.8031 & 0.9386 & 0.8943 & 0.9283 \\
\hline UIQI & 0.7992 & 0.8023 & 0.8019 & 0.9375 & 0.8834 & 0.9256 \\
\hline Mean & 120.23 & 120.13 & 119.01 & 197.56 & 195.473 & 197.12 \\
\hline Entropy & 7.5436 & 7.5439 & 7.5505 & 7.3784 & 7.4340 & 6.1434 \\
\hline S.D. & 54.378 & 55.4893 & 53.322 & 93.1467 & 94.904 & 94.213 \\
\hline SSIM & 0.6344 & 0.6211 & 0.68322 & 0.4355 & 0.4565 & 0.5644 \\
\hline
\end{tabular}



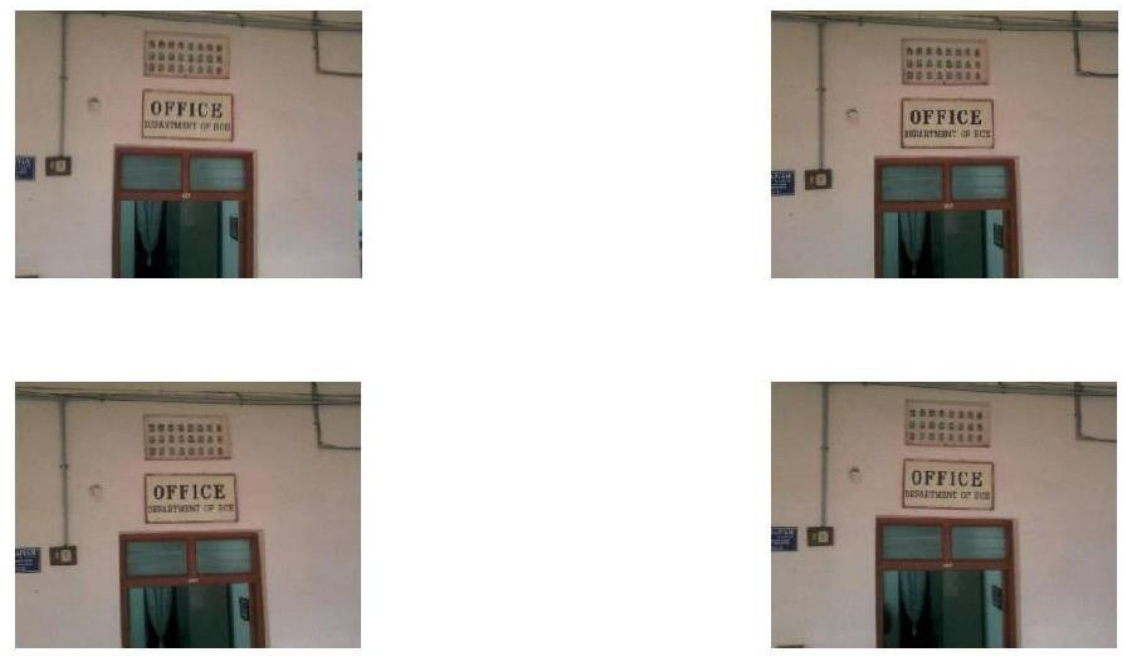

Figure 8. Low Resolution Dataset-1
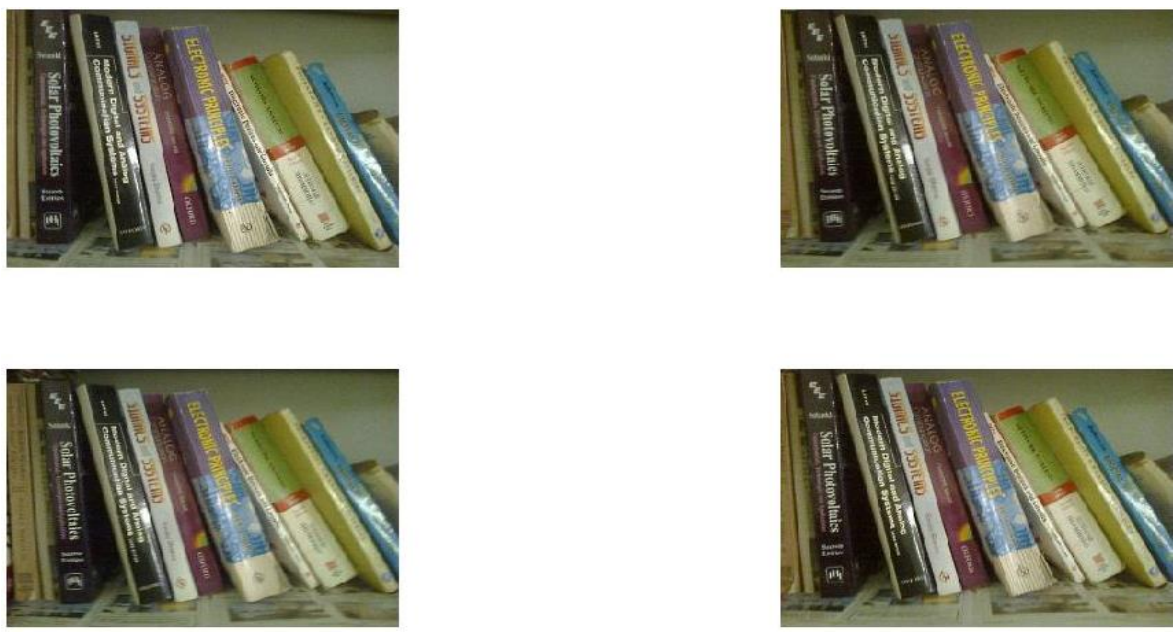

Figure 9. Low Resolution Dataset-2

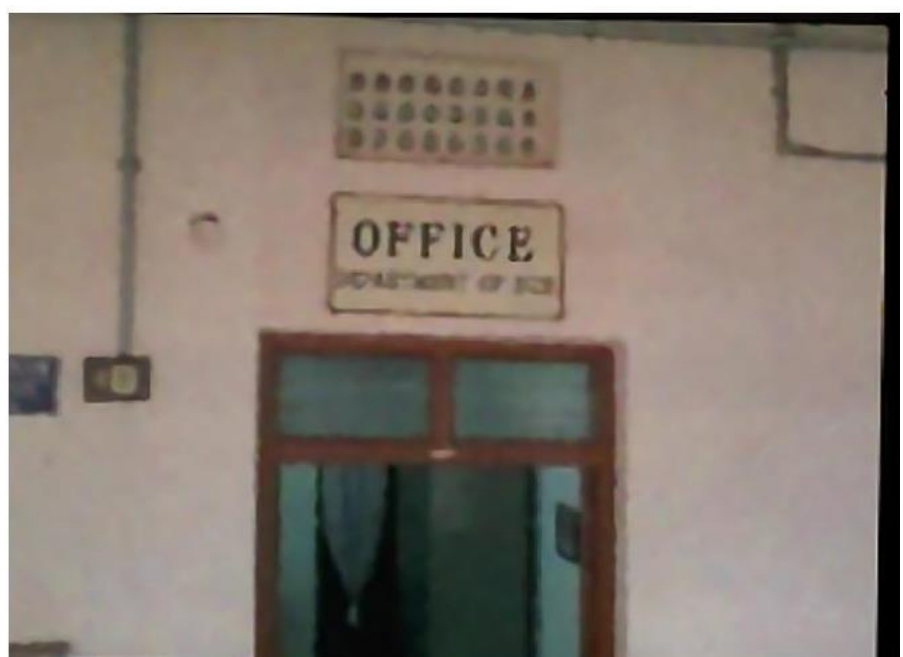

Figure 10. Restored Output of Blind Deconvolution based SR (Dataset-1) 


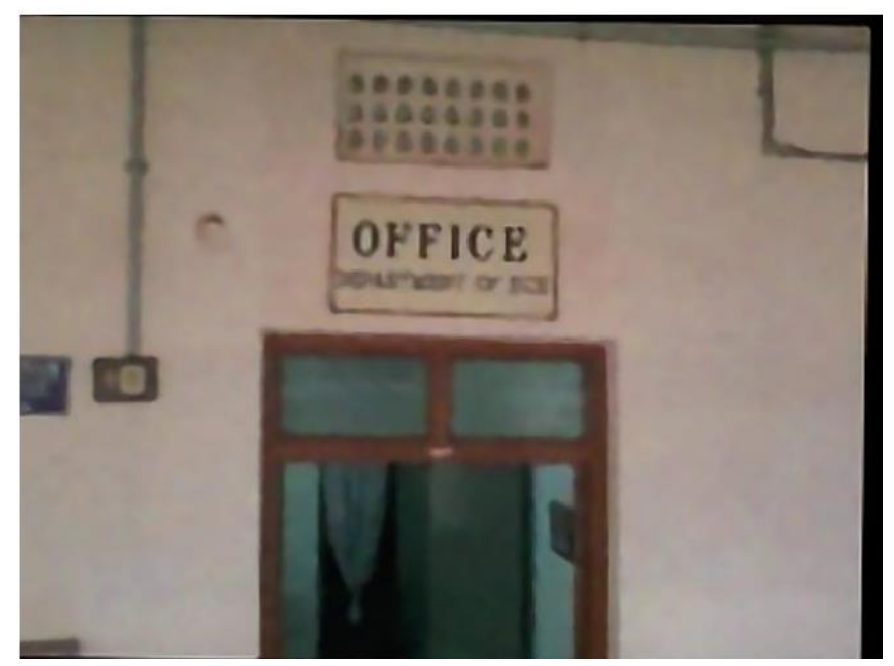

Figure 11. Restored Output of Adaptive Sharpening Filter based SR (Dataset-1)

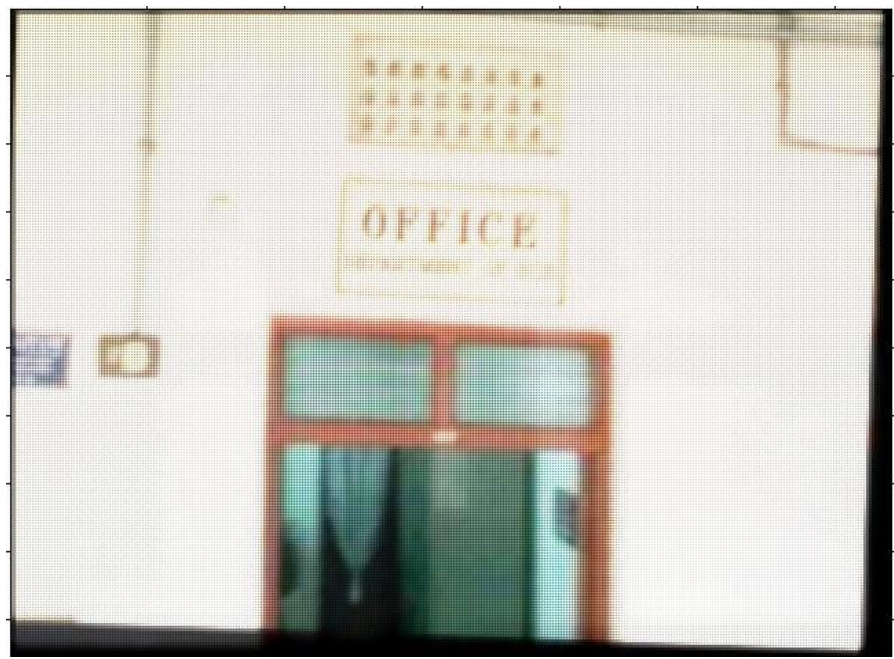

Figure 12. Restored Output of Proposed SR Algorithm Using Fuzzy based Bicubic Interpolation (Dataset-1)

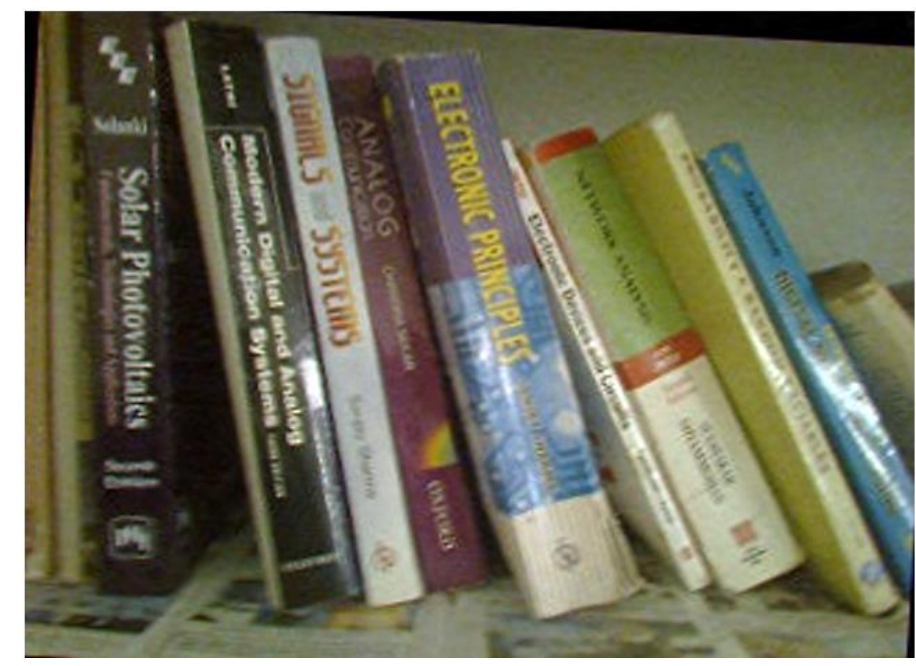

Figure 13. Restored Output of Blind Deconvolution based SR (Dataset-2) 


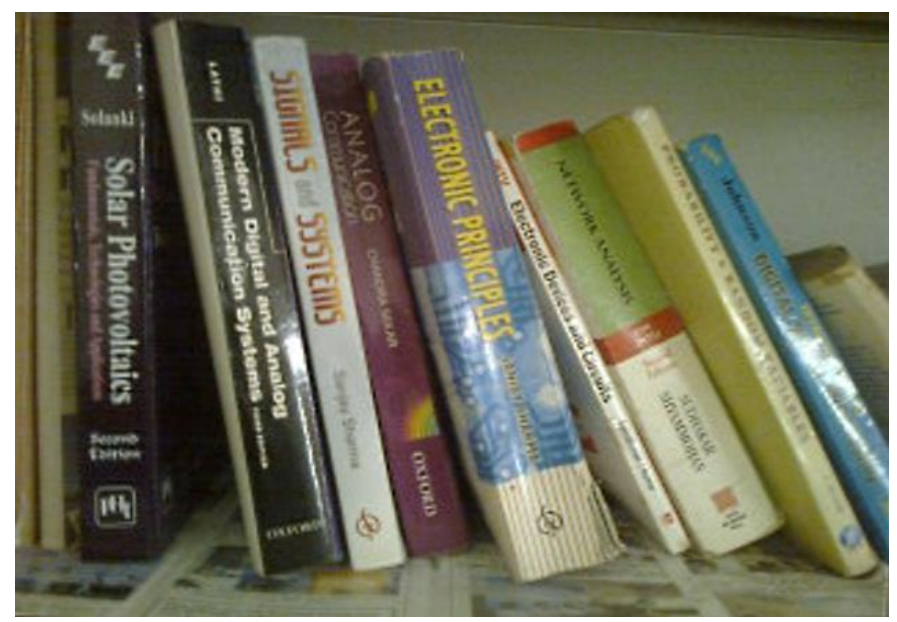

Figure 14. Restored Output of Adaptive Sharpening Filter based SR (Dataset-2)

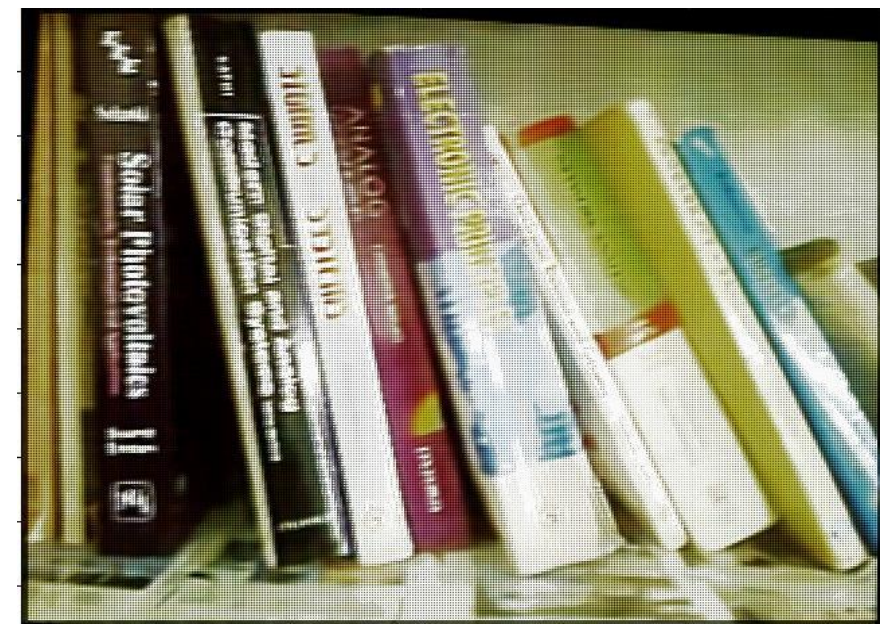

Figure 15. Restored Output of Proposed SR algorithm using Fuzzy based Bicubic Interpolation (Dataset-2)

\section{Conclusions}

A novel and efficient interpolation technique has been developed based on fuzzy theory concepts and applied to the SR reconstruction process to obtain the HR image. The algorithm is compared with the other results clearly demonstrate the improvement in terms of image quality and PSNR. The algorithm enhances the color information. Hence, the algorithm can be utilized in various applications like surveillance, medical and forensic sciences to effectively improve the resolution and enhance the chrominance information of the color images. The speed of operation decreases with the dimensions of the image. Hence, an algorithm with increased speed of operation has to be developed.

\section{References}

[1] S. C. Park, M. K. Park and M. G. Kang, "Super Resolution Image Reconstruction: A Technical Overview", IEEE Signal processing magazine, vol. 20, no. 3, (2003) May, pp. 21-36.

[2] M. Irani and S. Peleg, "Improving resolution by image registration CHIP: Graph", Models Image Processing, vol. 53, no. 3, (1991) May, pp. 231-239,

[3] L. Brown Gottesfeld, "Survey of Image Registration techniques", ACM Computing Surveys, vol. 24, no. 4, (1992), pp. 325-376. 
[4] H. Nasir, V. Stankovic and S. Marshall, "Singular Value decomposition based Fusion for super resolution Image Reconstuction Signal Processing: Image Communication”, vol. 27, (2012), pp. 180191.

[5] J. Hwang and H. Lee, "Adaptive image interpolation based on local gradient features", IEEE Signal Process, Lett., vol. 11, no. 3, (2004) March, pp. 359-362.

[6] C. Chang, Y. Chou, Y. Yu, and K. Shih, "An image zooming technique based on vector quantization approximation,” Image Vis. Comput., vol. 23, no. 13, (2005) November, pp. 1214-1225.

[7] J. Chen, J. Chang, and K. Shieh, "2-D discrete signal interpolation and its image resampling application using fuzzy rule-based inference", Fuzzy Sets Syst., vol. 114, no. 2, (2000) September, pp. 225-238.

[8] H. Ting and H. Hang, "Edge preserving interpolation of digital images using fuzzy inference," J. Vis. Commun. Image Represent., vol. 8, no. 4, (1997) December, pp. 338-355.

[9] R. Keys, "Cubic convolution interpolation for digital image processing,"IEEE Trans. Acoust., Speech, Signal Process., vol. ASSP-29, no. 6, (1981) December, pp. 1153-1160.

[10] H. Takeda, S. Farsiu, and P. Milanfar, "Kernel regression for image processing and reconstruction", IEEE Transactions on Image Processing, vol. 16, no. 2, (2007) February, pp. 349-366.

[11] Z. Xu, H. R. Wu, B. Qiu and X. Yu, "Geometric Features-Based Filtering for Suppression of Impulse Noise in Color Images", IEEE transactions on image processing, vol. 18, no. 8, (2009), August.

[12] A. G. Devi, T. Madhu and K. Lal Kishore, "An Improved Super Resolution Image Reconstruction using SVD based Fusion and Blind Deconvolution techniques", International Journal of Signal Processing, Image Processing and Pattern Recognition, http://dx.doi.org/10.14257/ijsip.2014.7.1.27, vol. 7, no. 1, (2014), pp. 283-298.

[13] A. G. Devi, T. Madhu and K. Lal Kishore, "Detection, Tracking and Identification of Moving Objects in a Video using Super Resolution - A Novel Approach", International Journal of Applied Engineering Research, ISSN 0973-4562, vol. 10, no. 3, (2015), pp. 5471-5487.

[14] A. Amanatiadis, I. Andreadis and K. Konstantinidis, "Design and Implementation of a Fuzzy Area Based Image-Scaling Technique", IEEE Transactions on Instrumentation and Measurement, vol. 57, no. 8, (2008) June, pp. $1504-1513$.

[15] D. G. Lowe, "Distinctive Image Features from Scale-Invariant Keypoint", International Journal of Computer Vision, vol. 59, no. 3, (2004) July, pp. 207-232.

[16] L. Yue, H. Shen, Q. Yuan and L. Zhang, "A locally adaptive L1_L2 norm for multi-frame superresolution of images with mixed noise and outliers", Signal Processing, vol. 105, (2014), pp. 156-174.

[17] M. Deshmukh and U. Bhosale, "Image Fusion and Image Quality Assessment of Fused Images", International Journal of Image Processing (IJIP), vol. 4, no. 5, (2010), pp. 484-508.

[18] K. S. Arun and K. S. Sarath, "An Automatic Feature Based Registration Algorithm for Medical Images", International Conference on Advances in Recent Technologies in Communication and Computing, IEEE Computer Society, (2010).

[19] Andreadis and A. Amanatiadis, "Digital image scaling," in Proc. IEEE Instrum. Meas. Technol. Conf, vol. 3, (2005), pp. 2028-2032.

[20] D. G. Lowe, "Object recognition from local scale-invariant features", International Conference on Computer Vision, Corfu, Greece, (1999) September, pp. 1150-1157.

[21] H. Nasir, V. Stankovic, and S. Marshall, "Image registration for super resolution using scale invariant Feature transform", belief propagation and random sampling consensus 18th European Signal Processing Conference (EUSIPCO-2010) (2010), August 23-27, Aalborg, Denmark.

[22] Z. Yuan. P. Yan, and S. Li, "Super resolution based on scale invariant feature transform", Proc. Int. Conf. Audio, Language and Image Processing, 2008, ICALIP 2008, 7-9 July (2008), pp. 1550-1554.

[23] T. Stathaki, "Image Fusion: Algorithms and Applications", First edition, Academic Press is an imprint of Elsevier, (2008).

[24] V. Bannore, "Iterative-Interpolation Super-Resolution Image Reconstruction-A Computationally Efficient Technique", Springer-Verlag Berlin Heidelberg, (2009).

[25] R. C. Gonzalez and R. E. Woods, "Digital Image Processing", $3^{\text {rd }}$ Edition, PHI, (2011).

\section{Authors}

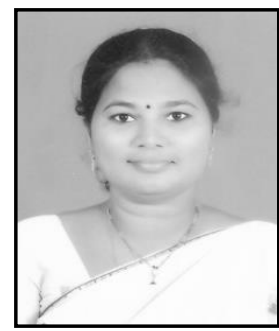

A. Geetha Devi, she is presently working as an Associate Professor in PVP Siddhartha Institute of Technology, Vijayawada, India. She received her $M$. Tech Degree from Pondicherry Engineering College, Pondicherry, India. Presently, she is pursuing her Ph.D. from Jawaharlal Nehru Technical University, Hyderabad, India under the guidance of Dr. T. Madhu and Dr. K. Lalkishore. Her research area is Image and Video Processing. 


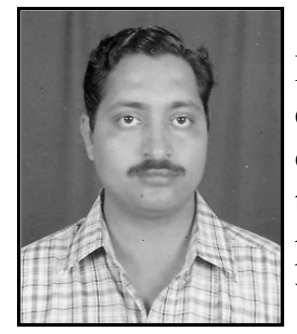

T. Madhu, he is currently working as a Principal at Swarnandhra Institute of Technology, Narasapuram, India. He received the Ph.D. degree from Osmania University, Hyderabad, India. He has 22 years of teaching experience. He guided several B. Tech and M. Tech theses. He has been guiding PhD students in the area of GPS, Image processing and Embedded systems. He published many papers in various National and International Journals.

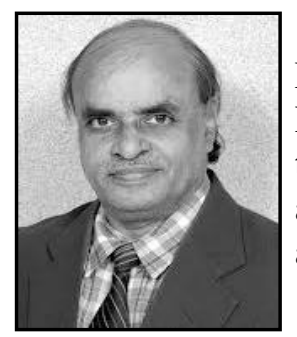

K. Lal Kishore, he is currently Vice Chancellor of Jawaharlal Nehru Technical University, Ananthapur, India. He guided several $\mathrm{PhD}$ students in the area of VLSI and Image processing. He has more than 100 publications in various National and International Journals and he is author of many books. His research interests are in VLSI and Image Processing 